\title{
Redução de seio frontal: abordagem bucomaxilofacial na terapia de reabilitação das fraturas panfaciais
}

\section{Frontal sinus reduction: approach oral and maxillofacial rehabilitation therapy of pan facial fractures}

\author{
Renato dos Santos* \\ Pâmela Marli Cavalheiro** \\ lara Fiorentin Comunello*** \\ Mara Campos ${ }^{* * * *}$ \\ Guilherme Luckmann ${ }^{* * * *}$
}

\section{Resumo}

Objetivo: relatar um caso clínico de fratura do seio frontal, com o manejo clínico e cirúrgico, as complicações e os benefícios dos tratamentos adotados. As lesões do seio frontal, na sua maioria, relacionam-se a traumas de grande amplitude, como acidentes com veículos automotores, agressões físicas, ferimentos com arma de fogo e acidentes em trabalhadores da construção civil, acometendo mais a população masculina entre 21 e 30 anos de idade, podendo afetar as paredes anterior e/ou inferior do seio frontal, incluindo fraturas naso-órbito-etmoidal e zigomáticas. O seu envolvimento pode causar complicações relacionadas a cavidade intracraniana, órbita e/ou estruturas nasais, como sinusites recorrentes, osteomielite do osso frontal, mucocele ou mucopiocele, meningite, encefalite, abcesso cerebral ou trombose do seio cavernoso, podendo evoluir para o óbito do paciente. Os objetivos do tratamento são: prevenção de infecção, isolamento do conteúdo intracraniano, correção da drenagem de líquido cefalorraquidiano, restauração da função e da estética, podendo ser realizado por equipe multidisciplinar. Relato de caso: o presente trabalho expõe um caso de fratura fronto-naso-órbito-etmoidal com afundamento de seio frontal, confirmada por exame tomográfico de face, em um paciente masculino de 26 anos, vítima de acidente automobilístico, submetido a redução da parede anterior do seio frontal, imobilização e fixação interna rígida, com posterior redução fechada da fratura dos ossos nasais, tamponamento nasal anterior e contenção externa com micropore e gesso. Considerações finais: demonstrou-se com esse artigo a possibilidade de divergência de tomada de conduta das diferentes áreas envolvidas no tratamento e a importância do tratamento multidisciplinar.

Palavras-chave: Bucomaxilofacial. Fraturas. Seio frontal.

\section{Introdução}

Sabe-se que o trauma do seio frontal corresponde a $8 \%$ das fraturas faciais, tendo como principal etiologia os acidentes automobilísticos ${ }^{1}$. Para definir a abordagem, deve-se, primeiramente, classificar o tipo de fratura. Observa-se que a mais comum é a da lâmina anterior do seio frontal, embora os casos mais graves apresentem acometimento da lâmina posterior e/ou do assoalho, podendo envolver o ducto nasofrontal ${ }^{2}$. $\mathrm{O}$ envolvimento dessa estrutura pode causar complicações relacionadas a cavidade intracraniana, órbita e/ou estruturas nasais, como sinusites recorrentes, osteomielite do osso frontal, mucocele ou mucopiocele, meningite, encefalite, abcesso cerebral ou trombose do seio cavernoso, podendo evoluir para o óbito dos pacientes ${ }^{3}$.

Os objetivos do tratamento, dependendo do tipo de fratura, são: prevenção de infecção, isolamento do conteúdo intracraniano, correção da drenagem de líquido cefalorraquidiano, restauração da fun-

Especialista em Cirurgia e Traumatologia Bucomaxilofacial, Hospital São Vicente de Paulo (HSVP), Mestrando em Odontologia, Universidade de Passo Fundo (UPF), Passo Fundo, Rio Grande do Sul, Brasil.

Estudante de Odontologia, Universidade do Vale do Itajaí (Univali), Itajaí, Santa Catarina, Brasil.

Especialista em Cirurgia e Traumatologia Bucomaxilofacial, HSVP-CEOM, Passo Fundo, Rio Grande do Sul, Brasil.

Cirurgiã-dentista, Univali, Itajaí, Santa Catarina, Brasil.

*** Cirurgião-dentista, Universidade Regional Integrada do Alto Uruguai e das Missões (URI), Erechim, Rio Grande do Sul, Brasil. 
ção e da estética ${ }^{1-3}$. Existem várias técnicas para reparar o seio frontal, que dependem da extensão da lesão e da patência do ducto nasofrontal. Dentre elas, destacam-se curetagem da mucosa do seio, brocagem das paredes, redução aberta e fixação interna-estável da parede anterior do seio, obliteração - caso o sistema de drenagem encontre-se envolvido - e cranialização, quando a parede posterior estiver envolvida ${ }^{4}$.

O presente trabalho expõe um caso de fratura de seio frontal com comprometimento da lâmina anterior associado à fratura panfacial, sem lesão da lâmina posterior ou de invasão intracraniana. Por conseguinte, a abordagem bucomaxilofacial é imprescindível para redução estética e anatômica da lesão, ossificação e prevenção das complicações possíveis $^{2-5}$. Desse modo, é fundamental a discussão sobre a possibilidade de uma abordagem multidisciplinar sempre que houver um caso de fratura de seio frontal. Assim, o objetivo do presente trabalho é relatar um caso clínico de fratura do seio frontal, com o manejo clínico e cirúrgico, as complicações e os benefícios dos tratamentos adotados.

\section{Revisão de literatura}

Os primeiros sinais de desenvolvimento do seio frontal estão presentes por volta dos quatro meses intrauterinos. Os recessos formados durante esse período terão algumas invaginações no osso frontal e no osso etmoide, sendo penumatizados no osso frontal, iniciando o desenvolvimento do seio frontal. A fisiologia de drenagem se dá por uma saída tipo óstio e, secundariamente, pelas células aéreas etmoidais. Radiograficamente, o seio frontal não é identificável até os 6 anos de idade ${ }^{5}$.

A pneumatização fica completa entre 12 e 16 anos de idade, mas pode continuar até os 40 anos. A altura média do seio frontal varia em aproximadamente $32 \mathrm{~mm}$, sendo sua largura média em torno de $26 \mathrm{~mm}^{6}$.

Os rebordos supraorbitários e a região anterior e inferior do osso frontal são as estruturas mais densas e resistentes. No centro, apresenta uma parede mais tênue, delgada e frágil. A parede posterior do seio frontal é mais delgada e se comunica com a cavidade intracraniana e com a base do crânio. $\mathrm{Na}$ porção póstero-medial do assoalho do seio frontal, localiza-se o trato de drenagem nasofrontal dado pelo óstio.O osso frontal é suprido pelas artérias supraorbital, temporal superficial anterior, cerebral anterior e meníngea média. Na região associada ao seio frontal, o suprimento ocorre pela artéria etmoidal anterior e por ramos da artéria esfenopalatina ${ }^{5}$.

A drenagem venosa é transóssea, por meio da anastomose dos vasos das estruturas subcutâneas, orbital e intracraniana. A drenagem venosa primária é feita pelos seios supratroclear, supraorbital, temporal superficial, intracraniano e dentro das veias subcutâneas ${ }^{6}$.

A inervação do seio frontal é dada pelo V2 nasal lateral posterior superior, assim como ramificações de VI do nervo etmoidal anterior. O seio frontal é o único que tem atividade retrograda de muco com movimento superior ao longo da parede medial. A função sinusal normal é mantida pelo epitélio respiratório coberto por uma camada de mucina, dependendo de drenagem adequada, a qual pode ser prejudicada por danos ao trato nasofrontal. Os cílios batem transportando secreções para o interior do meato médio do nariz; devido à sua localização, têm a função de proteção ao cérebro, agindo como uma barreira absorvedora de choque aos conteúdos intracranianos ${ }^{5}$.

O diagnóstico preciso das lesões do seio frontal é crucial para a realização de tratamento adequado. Os achados físicos sugestivos de fratura incluem abrasões na região frontal, lacerações, irregularidades no contorno ósseo, irritação, parestesias e hematomas ${ }^{7}$.

A tomografia computadorizada (TC) é o padrão-ouro para diagnóstico de fraturas do seio frontal. A imagem, vista pelo corte axial, fornece a melhor informação sobre a tábua anterior e a tábua posterior do seio frontal. Imagens tomográficas vistas pelo corte coronal são usadas para avaliar o assoalho do seio frontal e o teto da órbita. O exame visto pelo corte sagital pode ser útil na avaliação da permeabilidade do recesso frontal, sendo melhores do que em tomadas radiográficas bidimensionais ${ }^{7}$.

A decisão do tratamento adequado para a gestão das fraturas do seio frontal pode ser feita por avaliação de cinco parâmetros anatômicos, que incluem a presença de fratura de tábuas anterior e posterior, recesso nasofrontal, rompimento da dura (vazamento de liquor) e fratura com deslocamento/ cominutiva ${ }^{7}$. Com esses parâmetros, pode-se determinar o tratamento adequado. Dentre os tratamentos, destacam-se: observação, reparação endoscópica, redução aberta e fixação interna, obliteração do seio e cranialização sinusal ${ }^{8}$.

$\mathrm{O}$ acesso a todo o seio frontal é geralmente limitado pela fratura da tábua anterior. A exposição completa da tábua posterior é obtida por meio de uma craniotomia bifrontal. Os acessos cirúrgicos muitas vezes podem ser obtidos por uma laceração sobrejacente. Uma alternativa é o uso de incisões coronais, as quais proporcionam a exposição adequada do seio frontal e ficam camufladas pelos cabelos.

\section{Relato de caso}

Um paciente do gênero masculino, 26 anos, vítima de queda de veículo em movimento, foi levado à emergência do Hospital São Vicente de Paulo, na cidade de Passo Fundo, pela ambulância do muni- 
cípio de Ibiraiaras, em prancha rígida, com colar cervical e coxins laterais. Durante o atendimento inicial, contatou-se que ele não apresentou perda de consciência no momento do trauma, então, foi realizado protocolo estabelecido pelo suporte de vida avançado no trauma (ATLS) (Figura 1). No exame físico, constatou-se que o paciente demonstrava extenso ferimento corto-contuso, iniciando em região glabelar direita, com exposição óssea presente, estendendo-se até o dorso nasal e a asa nasal direita, com edema e hematoma periorbitário bilateral, ferimento corto-contuso em pálpebra superior esquerda, crepitações dos ossos nasais, sinais de epistaxe prévia autocontida, septo nasal preservado, musculatura extrínseca dos olhos preservada, acuidade visual preservada e pupilas isofotorreagentes. O paciente estava sem obstrução das vias aéreas, usava colar cervical, estava respirando e ventilando com normalidade, apresentava sangramento ativo em região glabelar direita, mas sem distúrbio circulatório. Estava lúcido, consciente, respondia a perguntas simples, tinha os reflexos motores preservados, tendo obtido pontuação 15 na escala de coma de Glasgow. Logo, foi submetido à limpeza dos ferimentos faciais e à sutura de contenção de sangramento facial sob anestesia local infiltrativa extranasal, seguida de controle regular dos sinais vitais e prescrição medicamentosa (Figura 2).

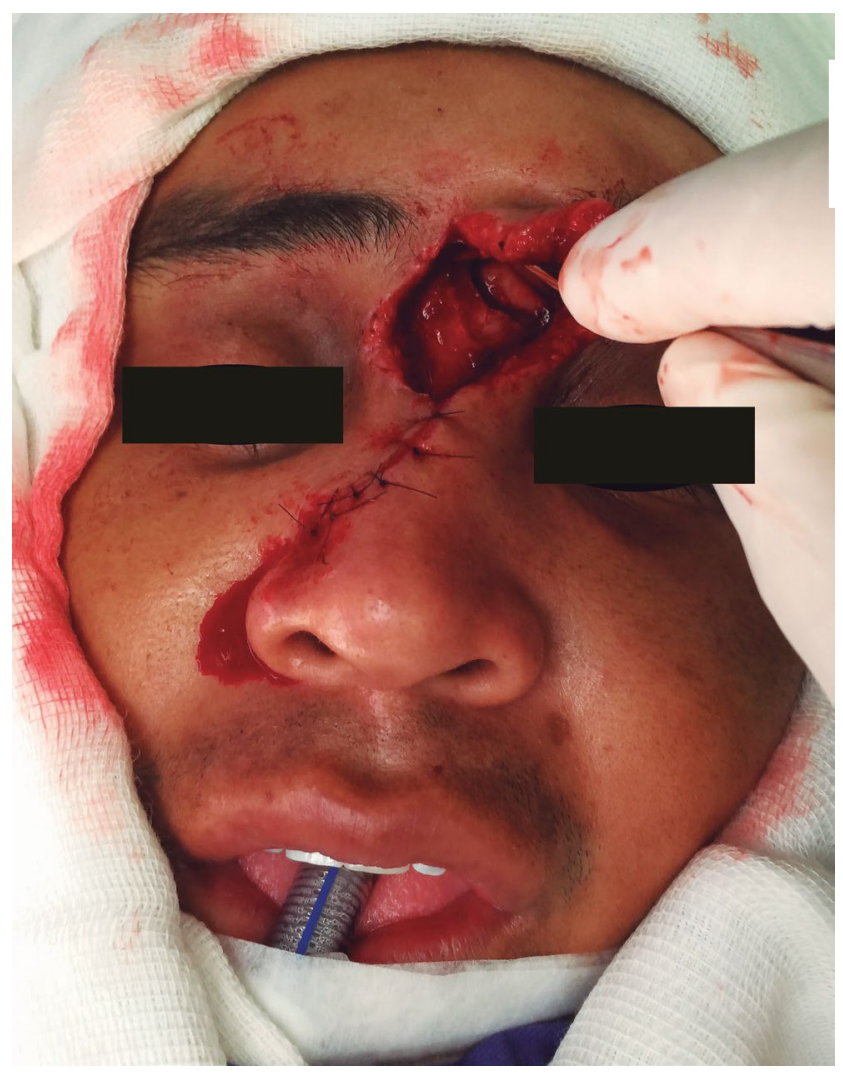

Figura 1 - Atendimento inicial com identificação de ferimentos corto-contusos na região facial

Fonte: acervo do autor.

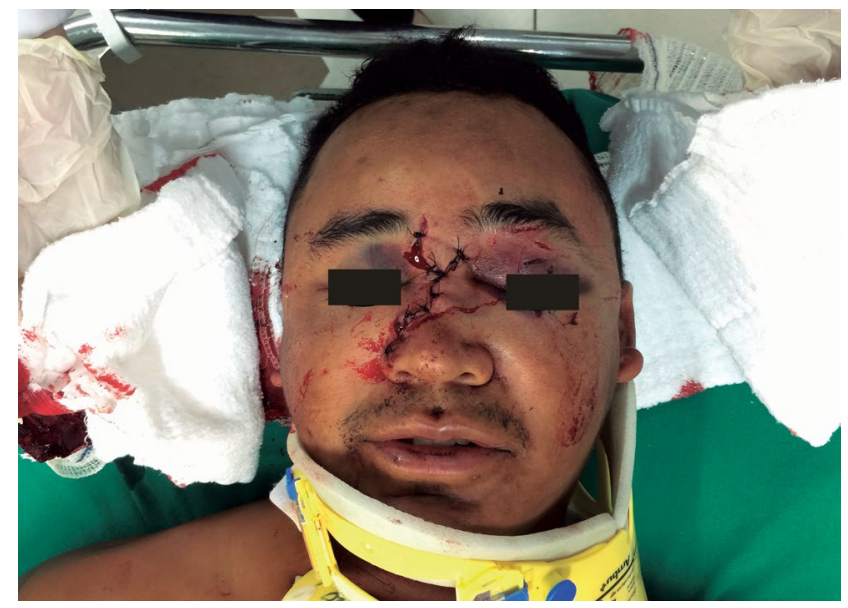

Figura 2 - Atendimento inicial com identificação de ferimentos corto-contusos na região facial

Fonte: acervo do autor.

Por intermédio do exame complementar por imagem tomográfico, constatou-se fratura fronto-naso-órbito-etmoidal com afundamento do seio frontal (Figura 3).

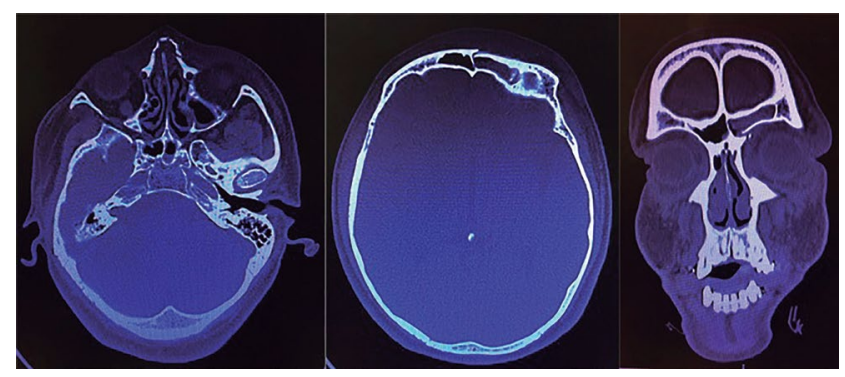

Figura 3 - Imagem tomográfica nos cortes axial e coronal mostrando a fratura do seio frontal

Fonte: acervo do autor.

Assim, optou-se por tratamento cirúrgico, com redução da parede anterior do seio frontal, imobilização e fixação interna rígida, com posterior redução fechada da fratura dos ossos nasais (Figura 4).

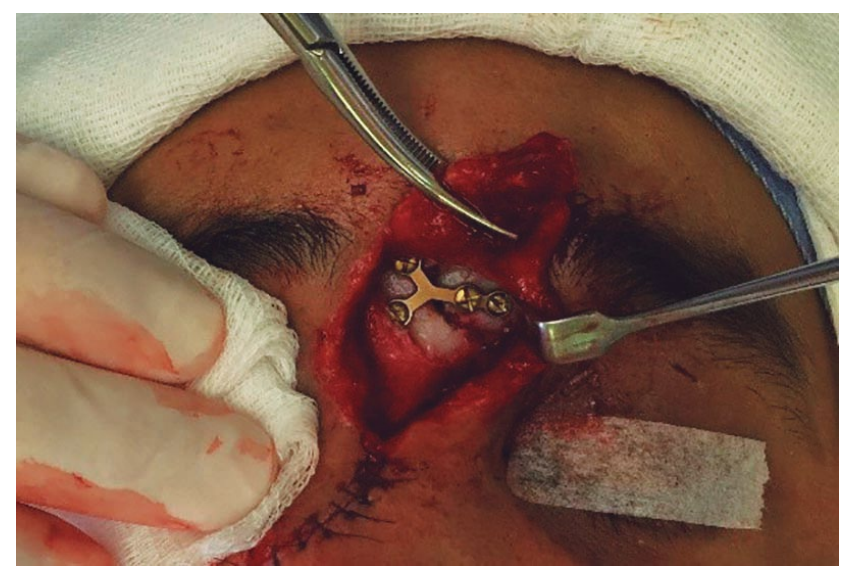

Figura 4 - Fixação interna rígida com sistema 1.5 (Synthes $($ ) e com escolha da placa em " $Y$ " para garantir estabilidade dos cotos

Fonte: acervo do autor. 
Para finalizar o ato cirúrgico, foi realizado o tamponamento nasal anterior e a contenção externa com micropore e gesso (Figura 5). Na prescrição medicamentosa pós-operatória, administrou-se antibiótico da classe das cefalosporinas de primeira geração (Cefazolina $1 \mathrm{~g}$ ), anti-inflamatório não esteroidal, analgésico periférico, analgésico opioide, protetor gástrico, antiemético, além de cuidados pós-operatório padrões, como manter a cabeceira elevada, não comprimir a região frontonasal, aspirar a orofaringe, quando necessário.

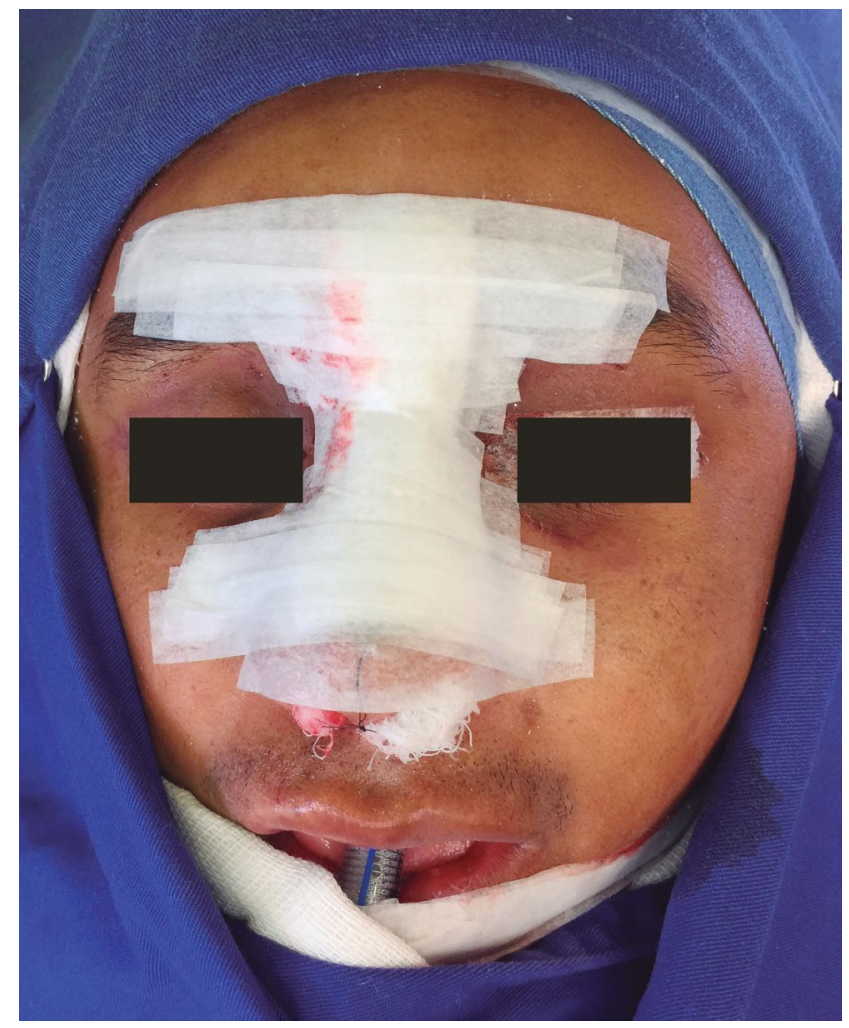

Figura 5 - Tamponamento nasal anterior com gazes e contenção gessada extranasal fixada com micropore

Fonte: acervo do autor.

Após a cirurgia, o paciente permaneceu com tamponamento nasal anterior e contenção externa durante dois dias. Após a remoção do tamponamento nasal anterior, iniciou-se a administração de Oximetazolina solução via nasal, no intervalo de 12 horas, para desobstrução nasal e controle de possíveis episódios de epistaxe, e de solução de cloreto de sódio administrado intranasal, no intervalo de 6 horas, para contribuir na lavagem dos seios paranasais. O paciente obteve boa evolução, com estabilidade dos sinais vitais, sem apresentar sinais flogísticos, algias ou complicações pós-operatórias.

Durante o período pós-operatório, o paciente relatou sensibilidade aos movimentos inspiratórios, com regressão espontânea em cinco dias, sem permanência de obstrução nasal (Figura 6).

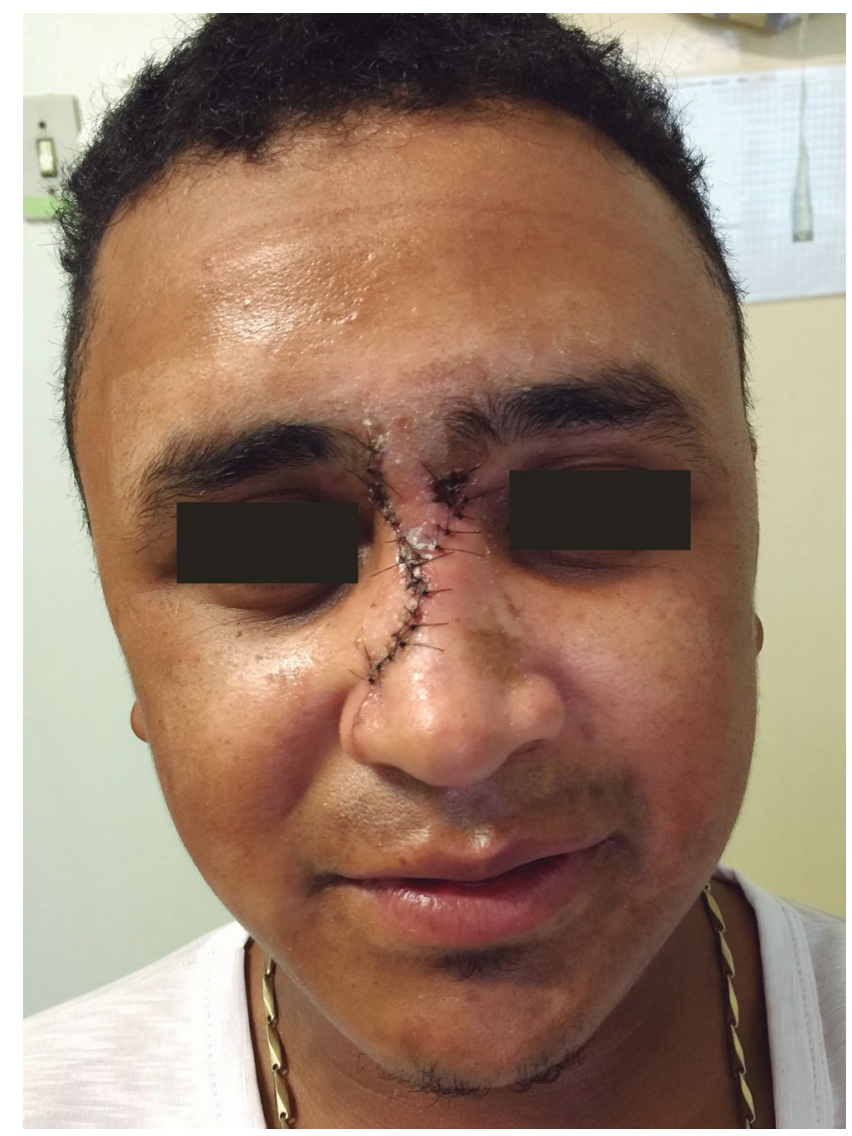

Figura 6 - Paciente em pós-operatório de 15 dias com suturas em posição sem sinais de fístula liquórica e infecção

Fonte: acervo do autor.

\section{Discussão}

A conduta clínica para as fraturas de parede anterior dos seios frontais não apresenta um consenso entre as especialidades, bem como existem diversas vertentes dentro da especialidade bucomaxilofacial. O mais relatado é que as fraturas em galho verde ou minimamente deslocadas não necessitam de tratamento cirúrgico. Deslocamentos mínimos, descritos como aqueles em que a quantidade de deslocamento é menor do que a espessura da tábua óssea vestibular, não necessitam de intervenção cirúrgica, exceto em casos em que ocorra um comprometimento estético que supere os riscos cirúrgicos envolvidos em qualquer procedimento $0^{9,10}$. Todavia, alguns autore $^{9,11}$ descrevem que deslocamentos mínimos devem ser tratados cirurgicamente, por meio de um acesso percutâneo, com a redução simples do afundamento da parede anterior, sem fixação, somente com realização de sutura em pele. A redução simples pode ser feita por meio de parafusos percutâneos, posicionados nos fragmentos e com consequente tracionamento dos fragmentos, posicionando os fragmentos em sua posição original, sem a necessidade de fixação ${ }^{10,12}$.

Fraturas deslocadas e/ou com múltiplos fragmentos requerem uma intervenção cirúrgica, para fins de evitar deformidades do contorno facial e, 
possivelmente, diminuir o risco de formação de mucocele, meningite, encefalite, abscesso cerebral ou sinusite $^{5,13,14}$. Os fragmentos devem ser reduzidos e fixados por sínteses, usando fios de aço número 0 , ou placas e parafusos de titânio sistema $1 \mathrm{~mm}$ ou $1,5 \mathrm{~mm}$. Caso o paciente apresente uma fragmentação excessiva da parede anterior, alguns profissionais sugerem a sua substituição por um enxerto ósseo, retirado de calota craniana ou do osso ilíaco.

A abordagem tradicional para os casos de fraturas consiste em incisões coronais e rebatimento dos tecidos, acesso por meio de ferimentos existentes devido ao trauma ou incisão direta sobre a região ${ }^{15}$. Há preferência pela utilização dos acessos por meio de ferimentos pré-existentes, porém, quando não existem, opta-se pelo acesso coronal, com a finalidade de reduzir defeitos estéticos no paciente. Outras técnicas também estão descritas na literatura, principalmente com utilização de endoscópios.

A utilização do endoscópio nas cirurgias para reparo de fraturas de seio frontal é amplamente discutida e estudada na área de cirurgia e traumatologia bucomaxilofacial, uma vez que possibilita ao cirurgião promover menores incisões e dissecamentos dos tecidos, sem que ocorra a diminuição da visibilidade pelo operador, em alguns casos, tem-se uma melhora na visibilidade. A realização de pequenas incisões promove resultados mais estéticos, diminuindo o tempo de internação do paciente e o tempo de recuperação pós-cirurgia ${ }^{16}$. Pesquisadores $^{14}$ relatam a utilização de telas de titânio fixadas por via endoscópica, permitindo um menor descolamento dos tecidos moles e reduzindo o tempo de internação hospitalar e os riscos de complicações pós-operatórias.

Entretanto, as técnicas para a utilização do endoscópio variam, principalmente pelo local de acesso. Arcuri et al. ${ }^{14}$ e Fattahi e Salman ${ }^{16}$ relatam a utilização de incisões paramedianas de 2-3 mm logo acima da linha capilar. Meiklejohn et al. ${ }^{17}$ relatam a utilização de incisões de $5 \mathrm{~mm}$ logo abaixo da porção medial da sobrancelha, acesso que pode ser empregado para reduções com ou sem a utilização de endoscópio. Também são empregados acessos transnasais para a utilização do endoscópio, evitando a realização de incisões externas ${ }^{12}$.

Outro acesso empregado para substituir a incisão coronal, descrito por Rao et al. ${ }^{18}$, é uma incisão logo abaixo da sobrancelha, compreendendo toda a sua extensão, permitindo o acesso à região da fratura e o descolamento tecidual que permite a posterior fixação por meio de telas e parafusos.

A drenagem e o debridamento do seio estão relacionados com presença ou não de fratura em assoalho do seio, com dano ao ducto nasofrontal ${ }^{9}$. Nos casos em que ocorra comprometimento do ducto, porém sem grande destruição das paredes do seio frontal, está indicada a realização da canulização do ducto nasofrontal. Já em casos de comprometimento do ducto nasofrontal e fratura das paredes do seio maxilar, indica-se a obliteração do seio frontal. $\mathrm{O}$ seio frontal deve ser acessado de modo que todo o seu interior seja debridado, promovendo a remoção dos tecidos de revestimento do seio e do ducto nasofrontal, e o espaço vazio formado deve então ser preenchido por um material compatível ${ }^{8,19}$. Dentre os materiais utilizados, destacam-se a gordura abdominal autógena, o enxerto ósseo osteoplástico ou o enxerto ósseo autógeno ${ }^{13,20}$.

\section{Conclusão}

Os traumas faciais, em geral, apresentam grande importância. O diagnóstico precoce e o tratamento adequado são imprescindíveis, devido à relação anatômica do seio com a fossa craniana anterior e o teto da órbita, além de elevada possibilidade de complicações, mesmo após vários anos do incidente traumático. Logo, a atuação multiprofissional é importante e o acompanhamento neurológico é imprescindível para os casos de fratura de seio frontal, pois suas complicações e sequelas podem ser fatais.

\section{Abstract}

Objective: to report a clinical case of frontal sinus fracture, clinical and surgical management, complications and benefits of the treatments adopted. Frontal sinus lesions, for the most part, are related to large-scale traumas such as accidents with motor vehicles, physical assault, gunshot wounds and accidents in construction workers, affecting more the male population between 21 and 30 years of age, and may affect the anterior and / or inferior wall of the frontal sinus including naso-orbito-ethmoidal and zygomatic fractures. Its involvement may cause complications related to the intracranial cavity, orbit and / or nasal structures such as recurrent sinusitis, osteomyelitis of the frontal bone, mucocele or mucopiocele, meningitis, encephalitis, cerebral abscess or thrombosis of the cavernous sinus, and may evolve to the death of these patients. The objectives of treatment are prevention of infection, isolation of intracranial content, correction of cerebrospinal fluid drainage, restoration of function and aesthetics, and can be performed by a multidisciplinary team. Case report: this paper reports a case of frontal-naso-orbito-orbito-ethmoidal fracture with frontal sinus sinking, confirmed by face tomography, in a 26-year-old male patient who had suffered an auto accident, submitted to a reduction of the anterior wall of the frontal sinus, immobilization and rigid internal fixation with posterior closed reduction of nasal bones fracture, anterior nasal packing and external restraint with micropore and gypsum. Final considerations: to demonstrate the possibility of divergence of the conduct of the different areas involved in the treatment and the importance of the multidisciplinary treatment.

Keywords: Maxillo-facial. Fractures. Frontal sinus. 


\section{Referências}

1. Araújo JCME, Nogueira FDFA, Lima Neto VNC. Cirurgia das fraturas do seio frontal: estudo epidemiológico e análise de técnicas. Rev Bras Otorrinolaringol 2006; 72:204-9.

2. Doonquah L, Brown P, Mullings W. Management of frontal sinus fractures. Oral Maxill Fac Surg 2012; 24(2):265-74.

3. Guy WM, Brissett AE. Contemporary management of traumatic fractures of the frontal sinus. Otolaryng Clinics 2013; 46(5):733-48

4. Ruggiero FP, Zender CA. Frontal sinus cranilization. Operat Techniq Otolaryng 2010; 21(2):143-6.

5. Fonseca RJ, Robert JW, H. Dexter Barber, Michael PP, $\square$ David EF. Trauma bucomaxilofacial. 4. ed. Rio de Janeiro: Elsevier; 2015.

6. Miloro M, Ghali GE, Larsen PE, Waite PD. Princípios de cirurgia bucomaxi-lofacial de Peterson. 2. ed. São Paulo: Santos; 2013.

7. Strong EB. Frontal sinus fracture. Operative Techniq Otolaryng 2008; 19:151-60.

8. Strong EB, Pahlavan N, Saito D. Frontal sinus fracture: a 28-year retrospective review. Otolaryngo - Head Nec Surg $2006 ; 135: 774-9$

9. Ioannides C, Freihofer AP. Fracture of the frontal sinus: classificarion and its implications for surgical treatment. Amer J Otolaryng 1999; 20(5):273-80.

10. Molendrijk J, Van der Wal KGH, Koudstall MJ. Surgical treatment of frontal sinus fractures: the simple percutaneous reduction revised. Int J Oral Maxillofac Surg 2012; 41:1192-4.

11. Manganelo-Souza LC, Luz JGC. Tratamento cirúrgico do trauma bucomaxilofacial. Rio de Janeiro: Roca; 2006.

12. Spinelli G, Lazzeri D, Arcuri F, Agostini T. Closed reduction of the isolated anterior frontal sinus frature via percutaneous screw placement. Int J Oral Maxillofac Surg 2014; 44(1):79-82.

13. Koudstaal MJ, Van der Wald KGH, Bijvoet HWC, Vincent AJPE, Poublon RMI. Post-trauma mucocele formation in the frontal sinus: a rationale of follow-up. Int J Oral Maxillofac Surg 2004; 33:751-4.

14. Arcuri F, Baragiotta N, Poglio G, Benech G. Post-traumatic deformity of the anterior frontal table managed by placement of a titanium mesh via an endoscopic approach. Brit J Oral Maxilllof Surg 2012; 50:53-4.

15. Tiwari P, Higuera S, Thornton J, Hollier LH. The management of frontal sinus fractures, J Oral Maxillofac Surg 2005; 63:1354-60.

16. Fattahi T, Salman S. An aesthetic approach in the repair of anterior frontal sinus fractures. Int J Oral Maxillofac Surg 2016; 45(9):1104-7.

17. Meiklejohn BD, Lynham A, Borgna SC. A simplified approach for the reductions of specific closed anterior table frontal sinus fractures. Brit J Oral Maxillofac Surg 2014; $52: 81-4$

18. Rao J, Blackburn TK, Clark S, Musgrove BT. Upper eyelid incision and use of a $90^{\circ}$ screwdriver for osteosynthesis of fractures of the anterior table of the frontal sinus. Brit J Oral Maxillofac Surg 2014; 51:974-5.

19. Jafari A, Nuyen BA, Salina CR, Smith AM, Deconde AS. Spontaneous ventilation of the frontal sinus after fratures involving the frontal recess. Amer J Otolaryng 2015; 36:837-42.
20. Vironneau P, Coste A, Oruliere-Escabasse V. Frontal sinus obliteration with autologous calvarial bone graft: indications and results. Eur Arch Otorhinolaryngol 2014; 271(11):2957-62.

Endereço para correspondência:

Renato dos Santos

Rua Bento Gonçalves, 1.568, Bairro Vergueiro 99020060, Passo Fundo, Rio Grande do Sul, Brasil Telefone: (54) 999538886

E-mail: dr.renatobucofacial@gmail.com

Recebido: 08/01/18. Aceito: 02/03/18. 